\title{
Gradhiva
}

Revue d'anthropologie et d'histoire des arts

19 | 2014

"L'Atlantique Noir » de Nancy Cunard. Negro Anthology 1931-1934

\section{Jacques Roumain et Nancy Cunard}

Poème de Jacques Roumain dédié à Nancy Cunard et lettre de Jacques

Roumain à Nancy Cunard, commentés par Léon-François Hoffmann

Léon-François Hoffmann et Jacques Roumain

\section{(2) OpenEdition}

Journals

Édition électronique

URL : http://journals.openedition.org/gradhiva/2810

DOI : $10.4000 /$ gradhiva.2810

ISSN : 1760-849X

Éditeur

Musée du quai Branly Jacques Chirac

Édition imprimée

Date de publication : 1 mars 2014

Pagination : 174-191

ISBN : 978-2-35744-073-9

ISSN : 0764-8928

Référence électronique

Léon-François Hoffmann et Jacques Roumain, « Jacques Roumain et Nancy Cunard », Gradhiva [En ligne], 19 | 2014, mis en ligne le 01 mars 2017, consulté le 19 avril 2019. URL : http://

journals.openedition.org/gradhiva/2810 ; DOI : 10.4000/gradhiva.2810 


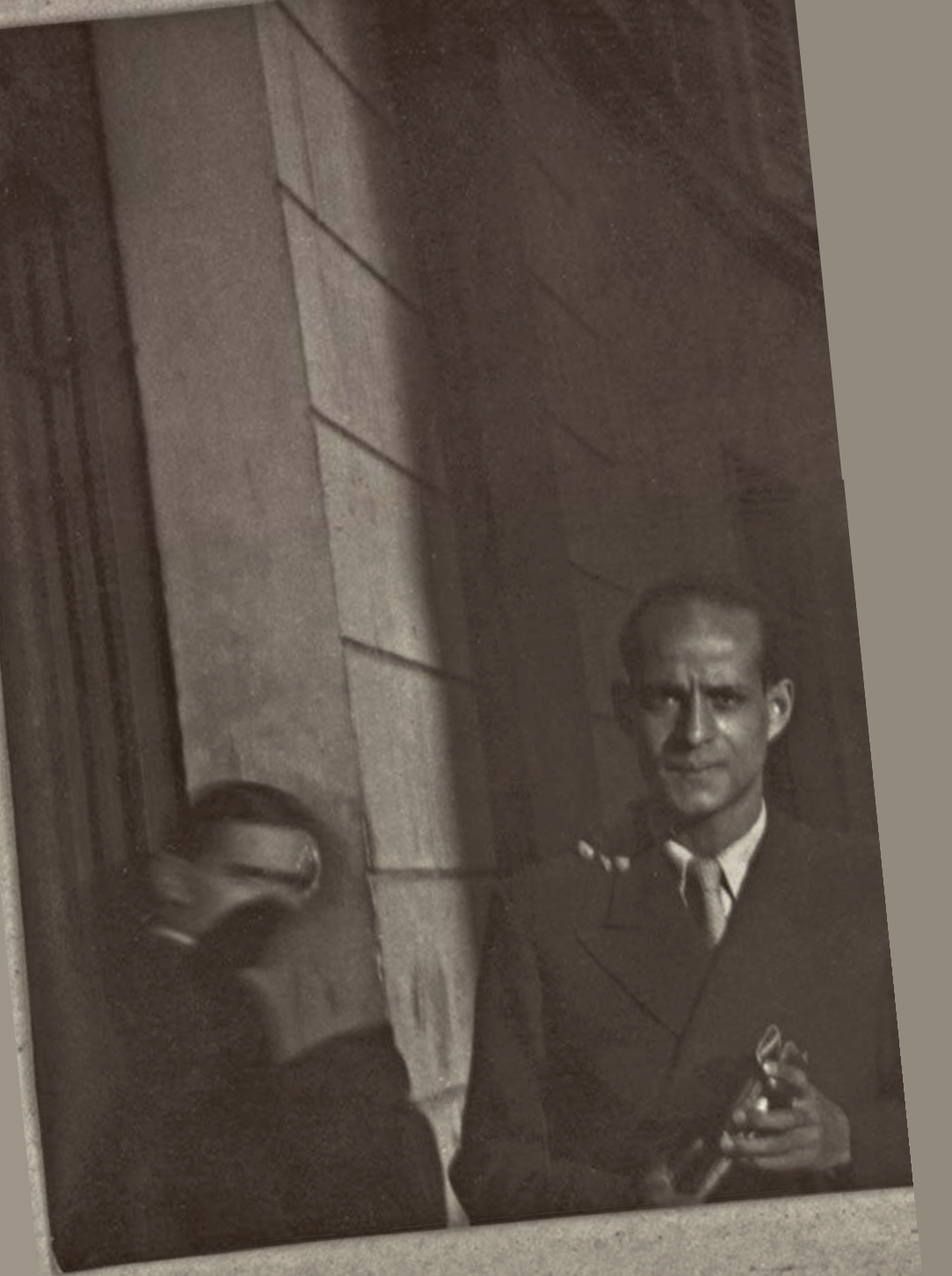




\section{Jacques Roumain et Nancy Cunard}

\section{Poème de Jacques Roumain dédié à Nancy Cunard et lettre de Jacques Roumain à Nancy Cunard, commentés par Léon-François Hoffmann}

\section{Cunard et Roumain}

L'exploitation des archives de Nancy Cunard par Sarah Frioux-Salgas a permis de mettre au jour plusieurs documents intéressant l'écrivain et homme politique haïtien Jacques Roumain (1907-1944) qui n'avaient pas trouvé place dans l'édition de ses CEuvres complètes ${ }^{1}$. II s'agit d'une lettre manuscrite adressée par lui à Cunard, de la traduction en anglais par celle-ci du texte de son intervention au Congrès international des écrivains pour la défense de la culture à Paris les 16 et 17 juillet $1937^{2}$, des manuscrits de ses poèmes Madrid et Sale Nègre, du tapuscrit de Madrid et enfin du manuscrit et du tapuscrit de son poème le plus célèbre: Bois-d'ébène.

Roumain est né à Port-au-Prince le 4 juin 1907 dans une famille de grands propriétaires terriens appartenant à la meilleure aristocratie mulâtre. Son grand-père maternel, Tancrède Auguste, fut même président de la république d'Haïti en 1912-1913. II commence ses études au prestigieux collège congréganiste de Saint-Louis-de-Gonzague puis est envoyé les continuer en Suisse et en Espagne. II revient en 1927 dans son pays, pour lors occupé par les marines: les États-Unis avaient mis le pays sous tutelle depuis 1915. II se lance immédiatement dans la vie littéraire, fonde deux revues, publie ses premiers poèmes et ne tarde pas à s'engager dans la lutte pour la libération du territoire. Ses articles attaquent violemment les occupants (qu'il accuse d'avoir importé en Haïti le racisme anti-Noir qui règne dans leur pays) mais aussi les préjugés coloristes de sa propre caste, qui profite cyniquement de l'occupation, et surtout le gouvernement fantoche du président Louis Borno, à la botte des Américains.

En décembre 1928, Roumain et deux autres journalistes sont arrêtés pour délit de presse. Il est condamné à un an de prison. Libéré avant terme, il est de nouveau emprisonné en octobre 1929. À la chute de Borno, en 1931, il est nommé chef de division au ministère de l'Intérieur par le nouveau président, Sténio Vincent. La même année, il publie La Montagne ensorcelée, premier roman haïtien à prendre pour cadre le monde rural et pour personnages de simples paysans, qui inspirera pendant plus d'un demi-siècle le courant littéraire du «roman paysan haïtien».

L'idéologie de plus en plus révolutionnaire et progressiste d'un intellectuel qui critique sa classe sociale et s'érige en porte-parole des paysans et des ouvriers inquiète le pouvoir: en 1933, Roumain est de nouveau emprisonné pour «menées subversives». II ne tarde pas à être relâché et, avec deux camarades, publie l'année suivante Analyse schématique 32-34, manifeste qui marque la fondation du premier parti communiste haïtien et provoque l'arrestation immédiate de son auteur et sa condamnation à trois

\author{
1. Édition critique coordonnée \\ par Léon-François Hoffmann, \\ Nanterre, ALLCA XX \\ ("Archivos» 58), 2003 \\ 2. Plus exactement des \\ séances de clôture du congrès, \\ qui s'était déroulé à Valence et \\ dans d'autres villes espagnoles. \\ Son mauvais état de santé avait \\ empêché Jacques Roumain \\ de se rendre en Espagne.
}


3. Christine Laurière, "Croyances religieuses populaires en Haïti », Gradhiva 1, 2005, p. 249-253.

4. Sur Roumain et l'ethnologie, voir Christine Laurière, "Jacques Roumain, ethnologue haïtien ", L'Homme 173, 2005/1, p. 187-197.

5. Je remercie Monsieur Lucien Degas et Madame Sylvie Zaidman de m'avoir signalé la publication du roman dans L'Humanité. ans de prison. II est de nouveau libéré après un an de détention, souffrant d'un paludisme contracté en prison et dont il subira les crises récurrentes le reste de sa vie. Interdit de séjour dans son propre pays, il s'exile à Bruxelles en 1936 avec sa femme et leur fils Daniel, puis à Paris, où il collabore à des revues de gauche telles Regards, Commune et Les Volontaires. II s'inscrit à I'Institut d'ethnologie et y suit l'enseignement de Paul Rivet ${ }^{3}$.

À la déclaration de guerre, Roumain envoie sa famille en Haïti et trouve in extremis une place sur un bateau bananier en partance pour la Martinique. Toujours interdit de séjour par son ennemi Sténio Vincent, il trouve refuge à New York où il tente de continuer ses études d'ethnologie à l'université Columbia 4 . Peut-être de peur d'être inquiété par les autorités états-uniennes pour sa participation à des manifestations de la gauche américaine, il se rend à La Havane à la fin du mois de décembre 1940. II y est accueilli par son vieux camarade cubain, le poète Nicolás Guillén. Au printemps suivant, Élie Lescot ayant remplacé Sténio Vincent à la présidence d'Haïti, Roumain peut enfin retrouver les siens et sa patrie. II rencontre l'anthropologue Alfred Métraux et sa femme, et participe à leurs recherches sur les cultures autochtones de l'île et le vaudou. Il est ensuite nommé chargé d'affaires à Mexico par le président, qui craignait sans doute de voir l'écrivain reprendre les activités subversives qu'il avait plus ou moins négligées depuis son retour. Peut-être soupçonnait-on que les enquêtes de terrain de ce chercheur communiste seraient pour lui l'occasion d'endoctriner les paysans.

À Mexico, Roumain fréquente les exilés politiques européens, pour la plupart communistes, dont Anna Seghers, et le documente pour ses futures fictions ayant Haïti pour cadre (Karibische Geschichten, Drei Frauen aus Haiti), ainsi que le poète Pablo Neruda, alors consul général du Chili, qui avait édité avec Cunard en 1937 à Paris une série de fascicules réunis en un recueil intitulé Los poetas del mundo defienden al pueblo español. Cet ouvrage a été réédité à Séville en 2002 avec quatorze pages de «souvenirs de l'ineffable Nancy Cunard » (Recuerdo de la inefable Nancy Cunard) par le romancier espagnol Ramón Sender, autre participant au congrès de 1937.

La santé de Roumain se détériore rapidement et, le 6 août 1944, il rentre à Port-au-Prince où il meurt deux semaines plus tard. Gouverneurs de la rosée, le roman paysan qui lui assurera un renom international, est publié à Port-au-Prince l'année suivante par les soins de sa veuve et de son frère. Louis Aragon, qui avait rencontré Roumain très probablement pour la première fois au Congrès des écrivains, le publie en 1946 à Paris, aux Éditeurs réunis qu'il dirige; L'Humanité le fait paraître en feuilletons entre les 26-27 janvier et le 12 avril $1947^{5}$. II sera traduit en dix-sept langues.

\section{Langston Hughes}

C'était probablement grâce au poète noir américain Langston Hughes que Cunard avait pris contact avec Roumain. Hughes, qui évoque avec beaucoup d'humour dans son autobiographie de 1956 / Wonder as I Wander (traduit en français par Léon-François Hoffmann dans les CEuvres complètes, op. cit., p. 1626-1631) sa rencontre avec son confrère haïtien lors de son voyage en Haïti en 1931, traduisit le poème de Roumain Quand bat le tam-tam et le proposa à Cunard, qui l'accepta pour sa Negro Anthology. 
Lorsque Roumain est condamné à trois ans de prison, Hughes s'affirme "écrivain de couleur moi aussi", et fonde en 1935 avec Francine Bradley, autre amie de Roumain, un Committee for the Release of Jacques Roumain, «de loin le plus talentueux des hommes de lettres d'Haïti 6 ».

Roumain et Hughes étaient tous deux à Paris en juillet 1937 lors des sessions de clôture du Congrès des écrivains pour la défense de la culture, et c'est alors que les deux écrivains firent la connaissance de Cunard. En ce qui concerne Hughes:

Le romancier Louis Aragon m'avait présenté cet été à Nancy Cunard, avec qui j'avais correspondu lorsqu'elle préparait sa grande anthologie Negro, mais que je n'avais jamais rencontrée, bien qu'elle se fût déjà rendue en Amérique?.

Quoi qu'il en soit, Cunard ne revit jamais Roumain ni - pour autant que l'on sache - Hughes... à moins qu'il n'ait été parmi les amis noirs de Nancy qui vinrent en juillet 1941 la voir à bord du bateau où elle attendait en vain la permission de débarquer à New York avant de rentrer en Angleterre.

Roumain et Hughes se rencontrèrent pour la dernière fois à New York lors de l'exil de Roumain en 1939-1940: une réception en son honneur fut organisée au YMCA de Harlem le 15 novembre 1939; Hughes, Richard Wright et de nombreux autres intellectuels noirs y assistaient. Sept ans plus tard, Hughes traduira Gouverneurs de la rosée avec Arna Bontemps. À la mort de son ami, il avait composé l'élégie $A$ Poem for Jacques Roumain.

De son côté, Roumain intitule Langston Hughes un poème publié dans Haïti-Journal le 20 octobre 1931 (CEuvres complètes, op.cit. : 47). Dans le même quotidien, il avait écrit une Présentation de Langston Hughes où il le jugeait «le plus grand poète noir de l'Amérique » et ajoutait: «il n'est point, à mon sens, d'écrivain de sa race qui l'égale comme romancier » (ibid. : 635). II s'était proposé de publier une anthologie de traductions de poètes noirs américains, dont Hughes, bien entendu, mais le projet ne vit pas le jour.

Venus d'horizons très différents, Roumain, Hughes et Cunard, ainsi que le poète cubain Nicolás Guillén, qui assistait au congrès, partageaient à la fois une vision du monde où dominait l'indignation envers les abus dont les Noirs étaient victimes en Afrique comme en diaspora, et la conviction que les idéologies de gauche étaient les seules à pouvoir mener à un monde plus juste et plus fraternel.

\section{Lettre à Nancy Cunard}

Nous ne savons pas exactement quand Roumain et sa famille quittèrent définitivement Bruxelles pour Paris, mais ce document permet de déterminer que cette date était postérieure au 14 juin 1937.

Si Cunard avait déjà publié en 1934, sous le titre When the Tom-Tom Beats, une traduction anglaise du poème de Roumain Quand bat le tam-tam (dans Negro Anthology), celui-ci ne l'avait pas encore rencontrée puisqu'il lui écrit que, si elle devait se rendre à Bruxelles, il «serai[t] très heureux de
6. L'appel de Langston Hughes a été publié en France dans plusieurs périodiques de gauche, dont Commune.

7. Langston Hughes, I Wonder as I Wander, New York, Thunder's Mouth Press, 1986, p. 318, notre traduction. Anne Chisholm, la biographe de Nancy Cunard, se trompe donc Iorsqu'elle affirme que celle-ci "a rapidement lié amitié " [quickly made friends] avec Hughes lors de son séjour à Harlem en 1931 (Nancy Cunard, New York, Knopf, 1979, p. 180). 
Bruselles 14. ㅈ. 37

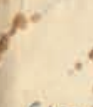

Chicer camarade Namen Cumard.

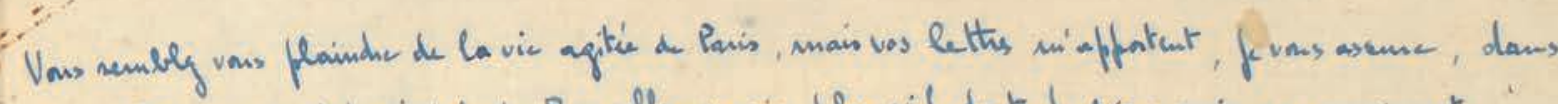

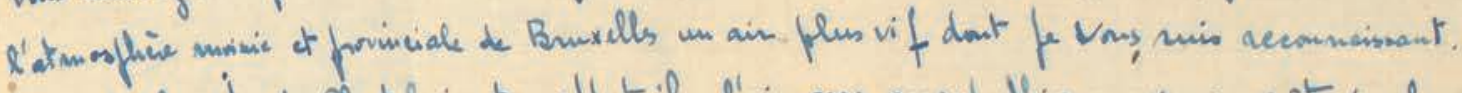

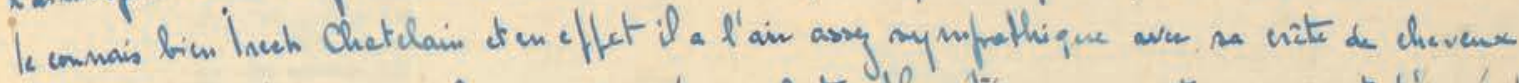

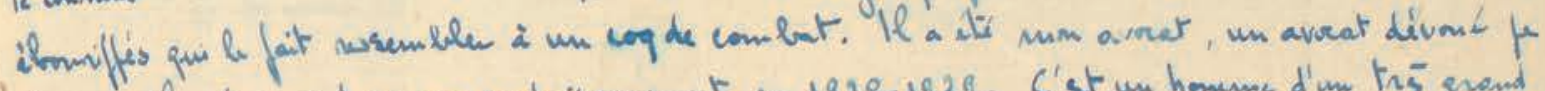

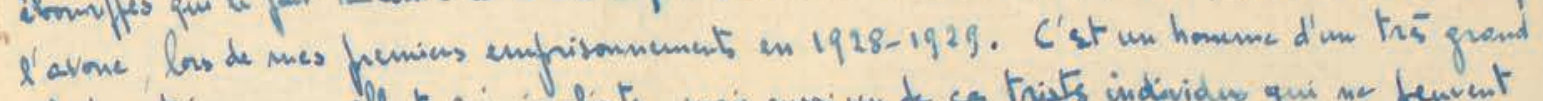

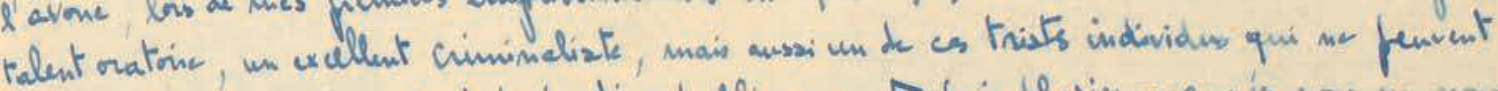

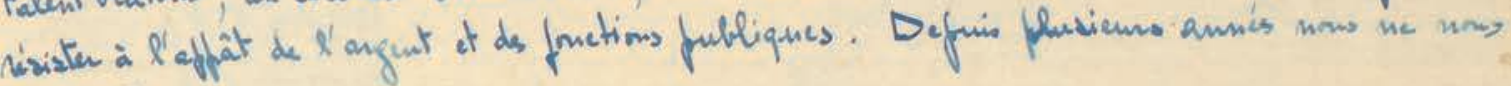

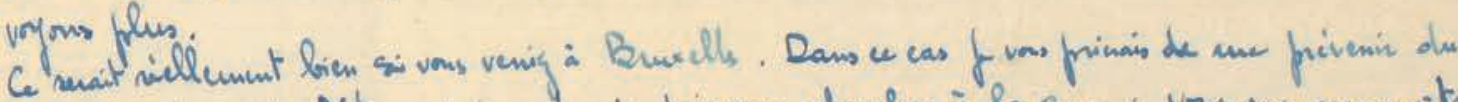

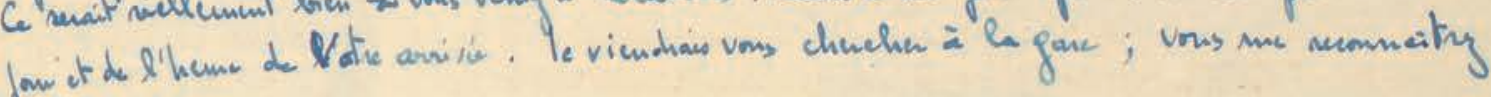
Cecilement à una conderne.

le serai the hemene de faine vithe connaissances.

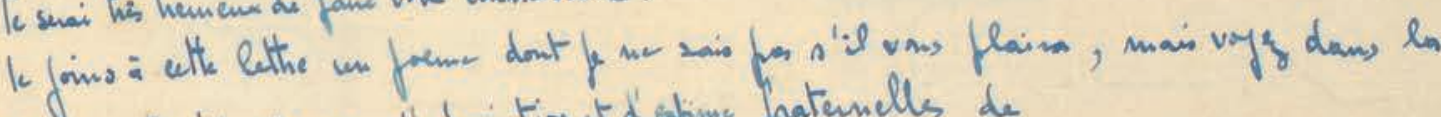

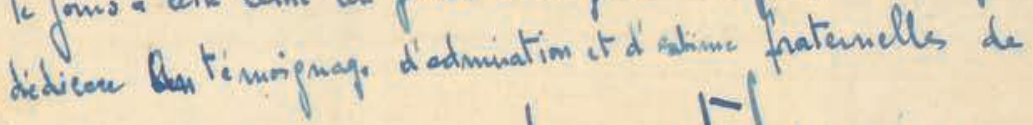

$$
\text { lacques| lorsuarer }
$$

Jacques Rouruain

"La Mondagme Ensorselé" 1931

"La Proie et e Ombre"

vhes fantoches.

BruXELLES

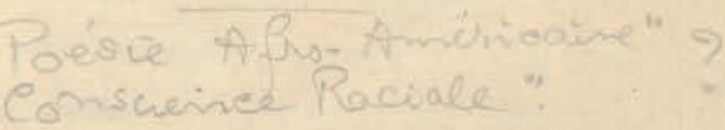


faire [sa] connaissance », et qu'elle le reconnaîtrait facilement à la gare «à [s] a couleur». Roumain appelle sa correspondante "Chère camarade Nancy Cunard ». Elle n'était jamais entrée au parti communiste, mais Roumain, fondateur du parti communiste haïtien, en savait assez long sur ses convictions pour l'appeler «camarade».

Roumain fait la connaissance de Cunard à Paris les 16 et 17 juillet 1937 lors des séances de clôture du Congrès des écrivains pour la défense de la culture qui avait réuni deux cents écrivains venus de vingt-huit pays, séances organisées au théâtre de la Porte-Saint-Martin par Louis Aragon et présentées par Robert Desnos en présence de Bertolt Brecht, André Chamson, Stephen Spender, Ilya Ehrenbourg et d'autres intellectuels antifascistes défenseurs de la cause républicaine espagnole. II y fit une intervention dont l'original ne nous est pas parvenu et qui ne fut, en tout état de cause, pas publiée dans le numéro de Commune consacré à cette manifestation, mais dont Cunard a publié une version anglaise dans «Three Negro poets $^{8}$ ". La traduction est vraisemblablement la sienne. Pour autant que l'on sache, Cunard n'a pas pris la parole au Congrès.

\section{Intervention de Roumain au Congrès}

Sous le titre «Three Negro poets», Cunard présente trois poètes noirs du continent américain dont elle a fait la connaissance au Congrès des écrivains: Hughes, Guillén et Roumain (qui se sont rencontrés pour la première fois à cette occasion et resteront amis). Elle les présente au lecteur, et fournit le texte de leurs interventions. Les extraits ci-dessous intéressent la personne et l'œuvre de Roumain:

[Au Congrès des écrivains] ont assisté trois délégués, trois militants, trois poètes de couleur. De couleur. Ce qui veut dire que pour eux le fascisme est un ennemi familier depuis la naissance - car qu'est-ce que l'impérialisme sinon le préjugé racial, les innombrables abus et injustices de la domination des Blancs? Tous trois ont pris la parole au Congrès, et comme chacun l'a affirmé, il est impensable pour un Nègre d'être autre chose qu'un antifasciste, car l'idéologie fasciste déverse insultes et humiliations sur les peuples de couleur. Si les noms de ces poètes ne sont pas encore connus en Angleterre, ils sont honorés - et craints - dans leurs pays respectifs: Langston Hughes, aux États-Unis, Nicolás Guillén, à Cuba, Jacques Roumain, en Haïti.

[...] Roumain a été arrêté sous l'accusation absurde d'avoir envoyé une bombe par la poste, mais en réalité [...] pour avoir élevé la voix en faveur des innocents de Scottsboro et des travailleurs noirs dans son propre pays. [...]

Roumain possède une profonde connaissance du patois haïtien, parlé dans toute l'île par les masses noires, composé de nombreux mots africains et d'une version plus ou moins phonétique du français, ainsi que du folklore haïtien dont les racines plongent en Afrique et qui reste aujourd'hui presque aussi purement nègre que l'arrièrepays encore culturellement indemne du continent noir. Haïti reste
8. Left Review, Londres, 3 octobre 1937, p. 535.

\section{ci-contre}

fig. 1

Lettre de Jacques Roumain à Nancy Cunard, 14 juin 1937. Harry Ransom Center. The University of Texas, Austin. Archives Nancy Cunard: Box 34/folder 5. D.R. 
9. Roumain n'a jamais, que l'on sache, travaillé pour le cinéma.
Haïti la Noire - malgré la pénétration impérialiste américaine.

[...] Roumain travaille au long scénario d'un film historique sur le temps de l'esclavage en Haïti ${ }^{9}$. Sa poésie est comme lui, délicate, forte et souple. C'est le plus modeste des hommes; il m'a même été difficile de me procurer son intervention; il ne l'avait pas écrite, il l'avait improvisée, et avait dit:

J'appartiens à une petite nation d'hommes noirs partisans de la liberté, qui ont joué un rôle important dans les luttes de libération du continent américain. Huit cents volontaires sont venus de mon pays participer à la Guerre d'Indépendance de l'Amérique du Nord et se sont courageusement battus à Savannah. Quand Bolivar, ayant essuyé une défaite temporaire, chercha refuge en Haïti, il trouva les armes et les munitions qui lui permirent de reprendre le combat. Cuba nous est redevable d'avoir porté assistance à Maceo et à Martí. Et, bien que la chose soit peu connue mais elle me semble néanmoins significative, le gouvernement haïtien de l'époque envoya d'importants secours financiers à la lointaine Grèce, aux champions de la liberté grecque qui luttaient pour l'affranchir du joug oppresseur de la Turquie.

Je suis fier également d'appartenir à ce même peuple d'esclaves noirs qui furent les premiers, il y a désormais cent trente-trois ans, à prendre les armes pour supprimer la tyrannie de leurs maîtres.

Je ne puis faire autrement que d'être un communiste, un antifasciste. Entre mille autres raisons, parce que je suis Nègre; parce que le fascisme condamne ma race à toutes les indignités.

En tant qu'écrivain, je m'engage pour la défense de la culture menacée par la barbarie fasciste. Ce qui implique que tout me pousse à faire miennes et les souffrances et la volonté de vaincre du peuple espagnol en lutte pour la liberté contre le fascisme, en lutte pour la dignité de l'espèce humaine. (Traduction de Léon-François Hoffmann, CEuvres complètes, op.cit., p. 680)

Comparée à d'autres interventions publiées dans Commune en octobre 1937, celles d'Aragon, par exemple, ou de l'Allemand Brecht, ou de l'Américain Hughes, ou de l'Espagnol Sender, ou encore du Cubain Guillén, entre autres, celle de Roumain (qui n'a pas été publiée par la revue) frappe par sa brièveté et par l'expression de son patriotisme et de son idéologie personnelle. II termine certes par l'affirmation de sa solidarité avec le peuple espagnol et de son appartenance au mouvement communiste, mais l'essentiel de son texte consiste en un rappel de l'histoire d'Haïti et de sa contribution aux mouvements de libération de plusieurs pays. Cette préoccupation didactique s'explique sans doute par l'ignorance, à l'étranger, de I'histoire, voire de l'existence même d'Haïti, ignorance générale à l'époque et loin d'avoir disparu aujourd'hui.

Les mots «noir» et «nègre» apparaissent trois fois dans le texte 
de Roumain: deux fois pour rappeler que les Haïtiens l'étaient, et une fois pour revendiquer sa propre appartenance à une race condamnée «à toutes les indignités». Cunard avait toujours affirmé sa sympathie et son attirance envers ce que l'on pourrait appeler - si ce n'était un anachronisme - la «négritude», vécue évidemment bien autrement par deux poètes noirs, Hughes, citoyen d'un pays placé sous le signe de la ségrégation tant officieuse qu'officielle, et Roumain, citoyen de la première république noire du Nouveau Monde.

Analyser en détail la place de la négritude dans l'œuvre de Roumain n'est pas notre propos. Elle a inspiré plusieurs réactions; la révolte, par exemple dans son poème Sales Nègres, qui s'ouvre par:

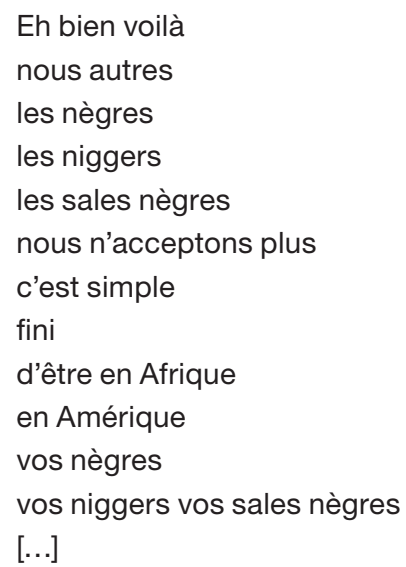

Son plus long et plus célèbre poème, Bois-d'ébène, se divise en deux parties; la première est une lamentation sur le sort des Africains déportés dans le Nouveau Monde sous l'appellation ironique de bois d'ébène, ou martyrisés au pays natal par les puissances colonisatrices, et la seconde, introduite par «CEPENDANT, je ne veux être que de votre race, ouvriers paysans de tous les pays", un appel à la fraternité de tous les travailleurs, les mineurs nègres de Johannesburg aussi bien que les mineurs blancs des Asturies, les ouvriers blancs de Détroit aussi bien que les péons noirs d'Alabama.

II faut aussi remarquer la présence de plusieurs tensions idéologiques chez l'écrivain. S'il a souvent dénoncé le racisme blanc, c'est surtout tel qu'il se manifestait aux États-Unis; or Roumain a passé quinze ans à lutter contre l'occupation de son pays par «le grand voisin du Nord». Il est difficile de savoir laquelle, des luttes pour la dignité raciale ou nationale, l'inspirait en priorité.

Mais plus encore qu'à l'occupant, c'est à sa propre caste bardée de préjugés et élite autoproclamée que Roumain réserve ses plus violentes attaques. Non seulement celle-ci exploitait sans vergogne le petit peuple de paysans et d'ouvriers, mais, constituée en majorité de ces mulâtres qui avaient pris la relève des Français à l'Indépendance, elle n'avait que mépris pour tout ce qui rappelait ses origines africaines, et le phénotype en particulier. Roumain avait bien écrit, dans l'Analyse schématique: «La couleur n'est rien, 
10. Lina Odena, citée à la deuxième ligne de l'avantdernier paragraphe du poème (voir fig.2), est une militante communiste née à Barcelone en 1911 qui, le 14 septembre 1936, choisit de se suicider, quelque part dans la province de Grenade, plutôt que de tomber entre les mains des troupes marocaines de Franco. De nombreux poètes ont célébré la mémoire de cette héroïne de la guerre d'Espagne.

\section{ci-contre}

fig. 2

Jacques Roumain, Tapuscrit du poème Madrid. Harry

Ransom Center. The University of Texas, Austin. Archives Nancy Cunard: Box 8/folder 5. D.R. la classe est tout" (CEuvres complètes, op.cit.: 656), il est difficile de savoir dans quelle mesure il souscrivait entièrement à ce mot d'ordre théorique.

Et enfin, sur le plan personnel, Roumain revendique constamment sa propre appartenance à la «race» noire. Son ami états-unien Hughes n'avait guère besoin d'en faire de même: son apparence suffisait, alors que Roumain était clair de peau et n'avait ni les cheveux crépus ni les traits négroïdes. Aucune trace dans ses écrits d'expériences personnelles de racisme ou même de discrimination lors de ses séjours aux États-Unis ou ailleurs. Se voulant Noir comme ses frères opprimés et pouvant passer pour Blanc comme leurs oppresseurs, Roumain a difficilement vécu cette situation. Quand bat le tam-tam, le poème que Hughes a précisément choisi de traduire pour l'anthologie de Cunard, se termine par ces vers:

Ton âme, c'est ce reflet dans l'eau murmurante où tes pères ont penchés [sic] leurs obscurs visages.

Ses secrets mouvements te mêlent à la vague Et le blanc qui te fit mulâtre, c'est ce peu d'écume rejeté, comme un crachat, sur le rivage.

(Ibid.: 44)

Cunard traduit ces deux derniers vers à la fin de sa présentation de Roumain et affirme que: "Ce n'est pas là de l'amertume raciste mais la revendication de ses droits humains du plus profond d'Haïti la Noire où un petit pourcentage de Blancs domine les millions de gens de couleur.»

Tous les textes de Roumain, en vers comme en prose, montrent comment l'écrivain a su articuler les diverses tensions qui l'habitaient pour forger une œuvre remarquable qui les transcende admirablement.

\section{Madrid}

Les archives de Cunard conservent un tapuscrit du poème Madrid ${ }^{\mathbf{1 0}}$, publié par Roumain dans la revue parisienne Commune en avril 1937 et, en tapuscrit également, sa traduction anglaise, «translated from the French of Jacques Roumain by Nancy Cunard ». Cette traduction n'a pas, à notre connaissance, été publiée. La version française ne diffère de la version publiée à Paris dans la revue Commune en avril 1937 (en même temps qu'un texte de Hughes et un poème de Federico García Lorca) que par deux variantes: "dans un petit square» qui devient «dans le petit square " et «l'âge de fer" qui devient «l'âge de feu», ainsi que par deux fautes de frappe corrigées.

On remarque cependant que la dédicace "À Ramón Sender» qui figure sur le document disparait et de la traduction, et de la version publiée. Sender (1901-1982) était un journaliste et romancier espagnol engagé volontaire dans les troupes républicaines. Les nationalistes assassinèrent sa femme et son frère. Après la défaite, il se réfugia en France, puis au Mexique. On ignore s'il y fréquenta Roumain lorsque ce dernier était chargé d'affaires d'Haïti à Mexico; probablement pas car, déçu vers la fin de la guerre par les exactions des commissaires politiques aux ordres du Komintern, Sender avait suscité leur méfiance. II n'est pas impossible que la rédaction 
(A Paru dans "COMMUNE"y Paris, Avril, 1937)

\author{
M A $\quad$ D $\quad R \quad I \quad D$ \\ à Ramón Sender \\ par Jacques Roumain
}

Cette ride sinistre de la sierra et l'horizon cerné d'une orage

le ciel $n^{\prime} a$ plus un sourire plus un seul tesson d'azur

pes un arc à lancer l'espoir d'une flèche de soleil

les arbres déchiquetés se redressent gémissant comme des violons desaccordés

tout un village endormi dens la mart d'en va à la dérive quand la mitrailleuse crible la passoire du silence

quand explose la cataracte de fracas

que le platras du ciel s'écroule

et les flammes tordues léchent dans la cite

les blessures des lézardes calfatées de nuit

et dans un petit square abandonné où règne maintenant la paisible

épouvante 11 y a

mais oui il y a sur le visage sanglant de cet enfant un sourire

comme une grenade écrasée à coups de talon.

Plus d'oisearax de doux chant d'oiseau des collines

l'age de fer et d'acier est né la siaison des sauterelles apocalyptiques

et les tanks avancent l'invasion obstinée de gros hannetons ravageurs

et I'homme est terré avec sa haine et sa joie pour demain

et quand il s'élance

la mort te vendange Hans Beimler

la mort qui agite sue le van de la plaine

une moisson de cris.

Voici avec la neige la denture carriée des montagnes

l'essaim des balles bourdonnant sur la charogne de la terre

et la peur au fond des entonnoirs est comme le ver dans une pustule

crevée

qui se rappelle l'incroyable saison le miel des vergers

et le sentier sous les branches

le murmure froissé des feuilles et le rire tendre et bon

1a paix du ciel et le secret des eaux -

de la jeune femme

I1 y a longtempo déjà que tomba dans l'oliveraie Lina Odena

18-bas dans le Sud.

C'est ici l'espace menacée du destin

la grêve où accourue de l'Atlas et du Rhin

la vague confondue de la fraternité du crime déferle

sur l'espoir traqué des hommes.

Mais c'est aussi malgré les sacré-coeurs brodés sur l'etendard

de Mahomet 
les scapulaires les reliques

les grigris duflucre

les fétiches du meurtre

les totems de l'ignorance

tous les vêtements du mensonge les signes démentiels du passé

ICI que l'aube s'arracke des lambeaux de la nuit

que dans l'atroce parturition et l'humble sang anonyme

du paysan et de l'ouvrier

nait le monde où sera effacé du front des hommes

la flétrissure amère de la seule égalité du désespoir.

JACQUES ROUMAIN

Poète du Haiti. 
de Commune ait décidé en 1937 de supprimer la dédicace sur ordre du parti. Sender finit par émigrer aux États-Unis, où il passa le reste de sa vie.

Les archives de Cunard conservent une photographie datée de juillet 1937 montrant celle-ci entre Roumain et Sender, avec leurs signatures et celle de Hughes (peut-être l'homme impossible à identifier qui apparaît à gauche du cliché, fig. 5).

En novembre 1936, les nationalistes font le siège de Madrid; plusieurs attaques frontales ayant échoué, Franco ordonne le bombardement en novembre. Comme Roumain mentionne la mort de Hans Beimler ${ }^{\mathbf{1 1}}$ et que celui-ci était tombé le $1^{\text {er }}$ décembre, on peut supposer que le poème a été composé pendant le premier trimestre de 1937.

\section{Bois-d'ébène}

À sa lettre à Cunard, Roumain joint un manuscrit «dont je ne sais pas s'il vous plaira », Bois-d'ébène, le plus célèbre de ses poèmes, qu'il dédie à sa correspondante. Si elle a également conservé dans ses archives le manuscrit de Sale Nègre, ce n'est très probablement pas à ce dernier que Roumain fait allusion dans sa lettre, puisque Nancy y a ajouté au crayon: «reçu en sept. 1937 à Paris ", c'est- à-dire après leur rencontre au Congrès des écrivains.

Bois-d'ébène est publié pour la première fois à Port-au-Prince par l'imprimerie Deschamps en 1945, très probablement par les soins de la veuve et du frère de Roumain, huit ans après le manuscrit envoyé à Cunard. II est dédicacé, dans le recueil posthume auquel il donne son titre, à Francine Bradley, femme de Lyman Bradley, professeur de sciences politiques à l'université de New York, laquelle avait assuré le secrétariat du Committee to Free Jacques Roumain. Le couple avait hébergé Roumain à son arrivée à New York en août 1939 et lui avait servi de boîte aux lettres lorsqu'il soupçonnait que sa correspondance était interceptée par les services secrets haïtiens et états-uniens.

Le manuscrit envoyé à Cunard est d'autant plus précieux que c'est le seul existant à notre connaissance, et qu'il est daté de Bruxelles, juin 1937, confirmant que la date de 1939 qui apparaît dans la version publiée est erronée. II comporte un certain nombre de variantes, généralement de nature stylistique, par rapport à la version publiée, qui n'affectent ni la structure ni le message du poème: remplacement d'un mot ou d'un fragment de phrase, alinéa ajouté ou supprimé, etc. Une comparaison systématique des deux versions n'aurait pas sa place ici. On se bornera à relever deux fragments qui disparaissent de la version définitive:

1. Dans le paragraphe immédiatement avant POURTANT, et après "Comme un fétiche tutélaire au centre du village », le vers suivant est supprimé dans la version définitive:

Comme un goût de sang et de cendre sur la langue.

2. Dans le paragraphe qui commence par «Si le torrent est frontière» après «affirmant les cordillères", le passage suivant de la dernière page du manuscrit disparaît dans la version définitive:

et telle la course d'un orage
11. Communiste allemand, emprisonné quatre semaines à Dachau avant de s'en évader, Hans Beimler est l'auteur d'un des premiers ouvrages sur les camps de concentration nazis : Im Mörderlager Dachau: Vier Wochen unter den braunen Banditen, Moscou et Léningrad, Verlagsgenossenschaft Ausländischer Arbeiter in der UdSSR, paru en 1933 et deux ans plus tard en français sous le titre: Au camp d'assassins de Dachau: quatre semaines aux mains des bandits à chemise brune, Paris, Imprimerie centrale, Bureau d'édition.

\section{ci-contre}

fig. 3

Jacques Roumain, Tapuscrit du poème Madrid. Harry Ransom Center. The University of Texas, Austin. Archives Nancy Cunard: Box 8/folder 5. D.R. 


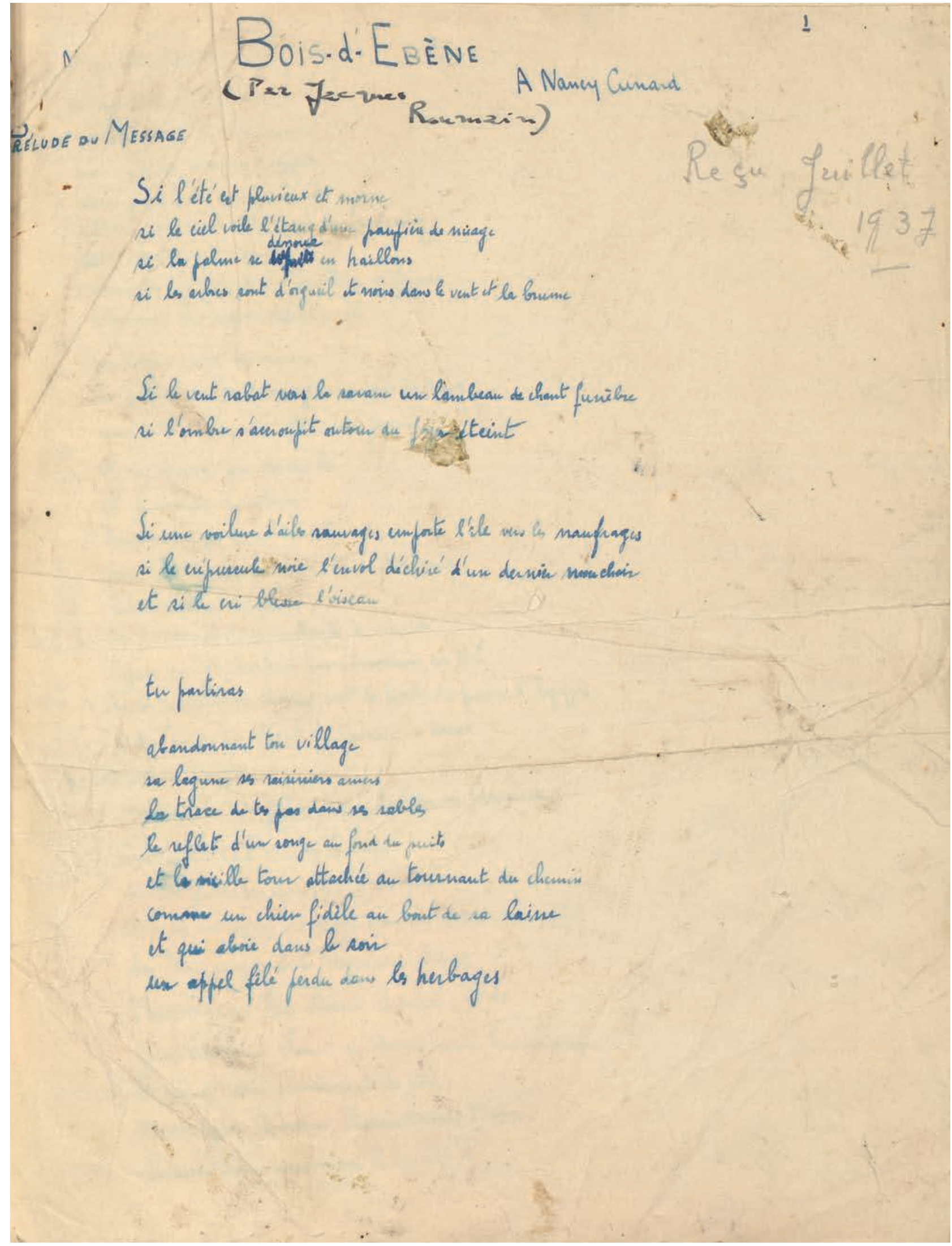


efface les nuages

d'un coup d'éponge

pour un ciel ébloui comme une page de soleil

la plaine sera l'esplanade d'aurore

BOIS-D’ÉBÈNE, extrait de Léon-François Hoffmann (coord.), CEuvres

complètes, op.cit., p.54-60.

À Francine Bradley

PRÉLUDE

Si l'été est pluvieux et morne

si le ciel voile l'étang d'une paupière de nuage

si la palme se dénoue en haillons

si les arbres sont d'orgueil et noirs dans le vent et la brume

Si le vent rabat vers la savane un lambeau de chant funèbre

si l'ombre s'accroupit autour du foyer éteint

Si une voilure d'ailes sauvages emporte l'île vers les naufrages

si le crépuscule noie l'envol déchiré d'un dernier mouchoir

et si le cri blesse l'oiseau

tu partiras

abandonnant ton village

Sa lagune et ses raisiniers amers

la trace de tes pas dans ses sables

le reflet d'un songe au fond du puits

et la vieille tour attachée au tournant du chemin

comme un chien fidèle au bout de sa laisse

et qui aboie dans le soir

un appel fêlé dans les herbages...

Nègre colporteur de révolte

tu connais tous les chemins du monde

depuis que tu fus vendu en Guinée

une lumière chavirée t'appelle

une pirogue livide

échouée dans la suie d'un ciel de faubourg

Cheminées d'usines

palmistes décapités d'un feuillage de fumée

délivrent une signature véhémente

La sirène ouvre ses vannes

du pressoir des fonderies coule un vin de haine

une houle d'épaules l'écume des cris

et se répand par les ruelles

et fermente en silence

dans les taudis cuves d'émeute

Voici pour ta voix un écho de chair et sang

noir messager d'espoir

car tu connais tous les chants du monde

\section{ci-contre}

fig. 4

Jacques Roumain, Manuscrit du poème Bois-d'Ebène, juin 1937. Harry Ransom Center. The University of Texas, Austin. Archives Nancy Cunard: Box 34/folder. D.R. 
12. Peuples de l'Afrique.

13. Ce mot n'existe pas Roumain avait-il écrit «hivers" ou «hévéas"?

14. Ligne de chemin de fer construite grâce au travail forcé des indigènes, dont la mortalité fut effrayante.

15. Allusion à la conquête de l'Éthiopie par les Italiens en 1935.

\section{page 174}

\section{et ci-contre}

fig. 5

Jacques Roumain, Nancy Cunard et Ramón Sender à Paris, 1937. Scrapbook de Nancy Cunard, "Cosas de España». Harry Ransom Center. The University of

Texas, Austin. Archives Nancy Cunard: box 27. depuis ceux des chantiers immémoriaux du Nil. Tu te souviens de chaque mot le poids des pierres d'Égypte et l'élan de ta misère a dressé les colonnes des temples Comme un sanglot de sève la tige des roseaux Cortège titubant ivre de mirages

Sur la piste des caravanes d'esclaves élèvent maigres branchages d'ombres enchainés de soleil des bras implorants vers nos dieux Mandingues Arada Bambara Ibo ${ }^{12}$ gémissant un chant qu'étranglaient les carcans (et quand nous arrivâmes à la côte Mandingues Bambara lbo quand nous arrivâmes à la côte Bambara lbo

il ne restait de nous

Bambara lbo

qu'une poignée de grains épars dans la main du semeur de mort) ce même chant repris aujourd'hui au Congo mais quand donc o mon peuple les hivées ${ }^{13}$ en flammes dispersant un orage d'oiseaux de cendre reconnaîtrai-je la révolte de tes mains? et que j'écoutai aux Antilles car ce chant négresse qui t'enseigna négresse ce chant d'immense peine négresse des îles négresse des plantations cette plainte désolée

Comme dans la conque le souffle oppressé des mers

Mais je sais aussi un silence

un silence de vingt-cinq mille cadavres nègres de vingt-cinq mille traverses de Bois-d'ébène

Sur les rails du Congo-Océan ${ }^{14}$

mais Je sais

des suaires de silence aux branches des cyprès des pétales de noirs caillots aux ronces de ce bois où fut lynché mon frère de Géorgie et berger d'Abyssinie

quelle épouvante te fit berger d'Abyssinie ce masque de silence minéral ${ }^{15}$

quelle rosée infâme de tes brebis un troupeau de marbre dans les pâturages de la mort

Non il n'est de cangue ni de lierre pour l'étouffer 
Ranuri 1. Senwer

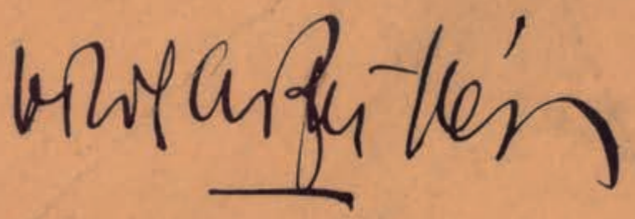

lacques I Iremair

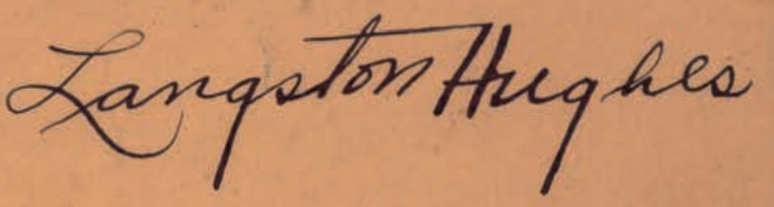

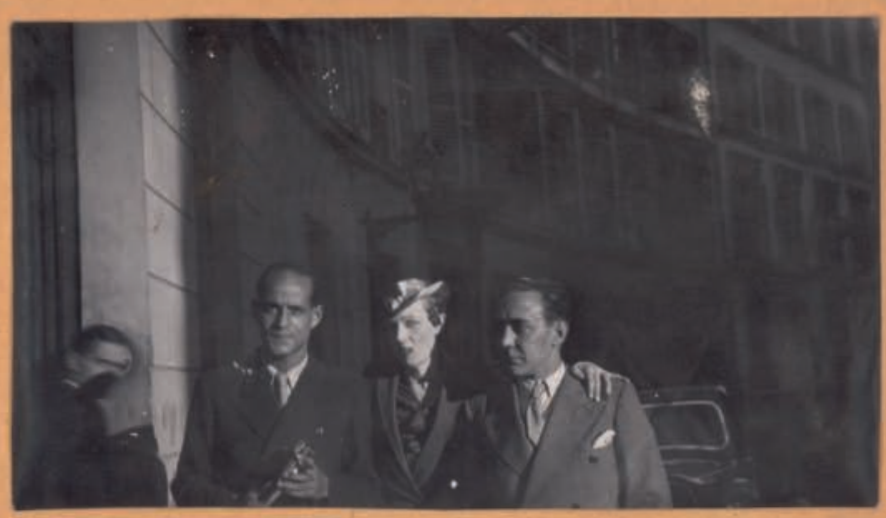

Jacques Roumzin, $\mathcal{N}$, Ramon Sende $r$

2) $P_{2} x$ is

Juilet I937 au moment du

Congaes des Ecxivains pout 12 Dejense

apres ses sessions en hispagne. 
de geôle de tombeau pour l'enfermer d'éloquence pour le travestir des verroteries du mensonge le silence

plus déchirant qu'un simoun de sagaies plus rugissant qu'un cyclone de fauves et qui hurle s'élève appelle vengeance et châtiment un raz-de-marée de pus et de lave sur la félonie du monde et le tympan du ciel crevé sous le poing de la justice

Afrique j'ai gardé ta mémoire Afrique tu es en moi

Comme l'écharde dans la blessure Comme un fétiche tutélaire au centre du village fais de moi la pierre de ta fronde de ma bouche les lèvres de ta plaie de mes genoux les colonnes brisées de ton abaissement...

\section{POURTANT}

je ne veux être que de votre race ouvriers paysans de tous les pays ce qui nous sépare les climats l'étendue l'espace les mers un peu de mousse voiliers dans un baquet d'indigo une lessive de nuages séchant sur l'horizon ici des chaumes un impur marigot là des steppes tondues aux ciseaux de gel Des alpages la rêverie d'une prairie bercée de peupliers le collier d'une rivière à la gorge d'une colline le pouls des fabriques martelant la fièvre des étés D'autres plages d'autres jungles l'assemblée des montagnes habitée de la haute pensée des éperviers d'autres villages est-ce tout cela climat étendue espace qui crée le clan la tribu la nation la peau la race et les dieux notre dissemblance inexorable? Et la mine et l'usine les moissons arrachées à notre faim notre commune indignité notre servage sous tous les cieux invariable? 
Mineur des Asturies mineur nègre de Johannesburg métallo de Krupp ${ }^{16}$ dur paysan de Castille vigneron de Sicile paria des Indes

(je franchis au seuil - réprouvé

je prends ta main dans ma main - intouchable)

garde rouge de la Chine soviétique ouvrier allemand de la prison de Moabit $\mathbf{1 7}$ indio des Amériques

Nous rebâtirons

Copan

Palenque

et les Tiahuanacos ${ }^{\mathbf{1 8}}$ socialistes

Ouvrier blanc de Detroit péon noir d'Alabama

peuple innombrable des galères capitalistes

le destin nous dresse épaule contre épaule

et reniant l'antique maléfice des tabous du sang

nous foulons les décombres de nos solitudes

Si le torrent est frontière

nous arracherons au ravin sa chevelure

intarissable

si la sierra est frontière

nous briserons la mâchoire des volcans

affirmant les cordillères

et la plaine sera l'esplanade d'aurore

où rassembler nos forces écartelées

par la ruse de nos maîtres

Comme la contradiction des traits

se résout en l'harmonie du visage

nous proclamons l'unité de la souffrance

et de la révolte

de tous les peuples sur toute la surface de la terre

et nous brassons le mortier des temps fraternels

dans la poussière des idoles

Bruxelles, juin $1939^{19}$
16. Famille allemande de fabricants d'armes.

17. Prison berlinoise où les nazis enfermaient et exécutaient leurs opposants, communistes et autres.

18. Anciennes villes de l'Amérique précolombienne.

19. Datation erronée, puisque Jacques Roumain avait quitté l'Europe le 27 mai et avait débarqué à la Guadeloupe le 8 juin, avant de rejoindre la Martinique, puis les États-Unis.

Peut-être faut-il lire 1937. 